\title{
NUMERICAL AND EXPERIMENTAL STUDY OF DAM-BREAK FLOOD PROPAGATION AND ITS IMPLICATION TO SEDIMENT EROSION
}

\author{
Hung-Chu Hsu ${ }^{1}$ A. Torres-Freyermuth ${ }^{2}$ Tian-Jian Hsu ${ }^{3}$ Hwung-Hweng Hwung ${ }^{4}$
}

\begin{abstract}
Regarding the hydrodynamics, within the past two decades it has become popular in numerical modeling of freesurface flow to adopt a Reynolds-averaged Navier-Stokes approach, where the volume of fluid (VOF) method is utilized to track the evolution of free-surface. However, this robust numerical model has not been widely applied to the study of sediment transport processes. In this study, we shall extend the numerical model to simulate suspended sediment transport and study the erosion pattern during the initial stage of the dam break flow. We also conducted a series of experiments in a horizontal channel of rectangular section and recorded the snap shots of surface profiles of a dam- break wave during the initial stage of dam-break. Measured data is utilized here to study the hydrodynamics and to validate the numerical model.
\end{abstract}

Keywords: numerical modeling; dam-break flow; laboratory tests; sediment transport

\section{INTRODUCTION}

Recently, heavy rains brought by typhoon Morakot in August 8, 2009 in Taiwan resulted in tens of landslide dams located in several counties in southern Taiwan (Water Resources Agency 2009). The rapid failure of landslide dam near Hsiao-Lin village causes great loss of lives. Although fluvial morphology is conventionally predicted by variation in stream power, it is unclear whether these episodic and catastrophic flooding events can be well described by the conventional approach developed and calibrated mostly for regular or seasonal variations (for an example on sediment discharge, see Kao and Milliman 2008). In order to quantify the characteristics of dam-break floods and its implication to sediment erosion in a well-controlled and repeatable environment, flume experiments aided with numerical modeling are adopted in this study.

Almost all the existing numerical modeling studies on dam-break flow are based on depthintegrated shallow water equations (Wu and Wang 2007; Abderrezzak et al. 2008). Although these models are computationally efficient to model large-scale river flooding and fluvial morphology. Major assumptions in these models, such as assuming hydrostatic pressure limits a more detailed process-based study on the initial stage of the damp-break flow. For instance, Standby et al. 1998 and Huang et al. 2010 confirmed that the wave breaking and turbulence dominate the flow field during the initial stage of dam-break waves and hence the assumptions of long wave and hydrostatic pressure using in the conventional theoretical and numerical model are not valid. Moreover, sediment transport under initial dam break flow may be significantly affected by break bore turbulence and hence more detailed modeling approaches that provide vertical structure of flow velocity and turbulence statistics are particularly useful. These key features, namely, the initial breaking waves, and its interactions with bottom sediment transport essentially need to be parameterized in the depth-integrated long wave model for large-scale prediction. We believe that only through a better understanding on the initial dam break flow and its effect of sediment transport through small-scale physical and numerical modeling efforts, appropriate parameterizations may be further developed.

Prior studies have been devoted to address the aforementioned limitations. For instance, Capart and Young 1998 and Fraccarollo and Capart 2002 developed a sediment transport module based on two-layer two-phase flow formulation for depth-integrated model (see also Cheng and Peng 2006). The sediment transport module is physically-based, where careful averaging procedure is performed and closures of intergranular interactions are incorporated. However, they are more applicable to transport of coarser grain where bedload is the dominant transport mechanism. On the other hand, for sediment transport of finer grain size (ratio of settling velocity to characteristic flow velocity, i.e., the fall parameter, is small), the suspended load dominates and a different modeling approach is required.

${ }^{1}$ Tainan Hydraulics Lab., National Cheng-Kung University, 5F., No.500, Sec. 3, Anming Rd., Annan District, Tainan City, 709, Taiwan

${ }^{2}$ Laboratorio de Ingenieríay Procesos Costeros, Instituto de Ingeniería, Universidad Nacional Autónoma de México, Sisal, Mexico

3 Center for Applied Coastal Research, Civil and Environmental Engineering, University of Delaware, Newark, DE 19716, USA

${ }^{4}$ Department of Hydraulic and Ocean Engineering, National Cheng-Kung University, No.1, University Road, Tainan City, 701, Taiwan 
In this study, we focus on resolving detailed hydrodynamics and suspended sediment transport processes using a two-dimensional-vertical (2DV) model based on Reynolds-averaged Navier-Stokes equations with k- $\varepsilon$ turbulence closure for free-surface flow. The overall aims of this paper are twofold. Firstly, we quantitatively analyze new experimental data on initial dam break flood propagation and validate the hydrodynamics of the numerical model. Secondly, we study the mechanism and pattern of sediment erosion due to different conditions of the dam break flow.

\section{EXPERIMENTAL SETUP}

The laboratory experiments were carried out in a wave flume at Tainan Hydraulics Laboratory, National Cheng Kung University. The flume is $26.59 \mathrm{~m}$ long, $0.37 \mathrm{~m}$ wide and $0.6 \mathrm{~m}$ in height. The schematic plot is shown in Fig. 1. The side walls are constructed with smooth glass, the lock and lock gate automatic controlled by air compressor are made from steel plate. The distance of the lock gate was fixed at $\mathrm{x}=11.27 \mathrm{~m}$ in the experiment and two initial upstream filling heights $(\mathrm{h})$ are chosen as 0.3 $\mathrm{m}$ and $0.4 \mathrm{~m}$. Coordinate $x$ refers to the position of the moving front of the dam-break flow, where the origin is fixed at the location of the lock gate. Four cases with different downstream water depths $(\mathrm{d}=0.015 \mathrm{~m}, 0.03 \mathrm{~m}, 0.06 \mathrm{~m}$ and $0.09 \mathrm{~m})$ were used to simulate dam break wave propagation. Table 1 summarizes all experimental conditions. The gate separating the reservoir from the rest of the tank and was removed from above at an approximately constant velocity (Vgate $=1.5 \mathrm{~m} / \mathrm{s}$ ). The experiments were record by one charged-coupled device (CCD) camera and 10 wave gauges. Furthermore, freesurface elevation time series, sampled at 10 locations with sampling $20 \mathrm{~Hz}$, provide high spatial and temporal resolution data of the water front propagation. The sensors are identified in this study with the acronym ' $\mathrm{WG}$ ' followed by the gauges number (i.e. WG1, WG2,..,WG10). A high-speed video camera with a frame acquisition rate of $3 \mathrm{~Hz}$ was utilized to qualitatively observe the wave front transformation during distinct dam break wave evolutionary stages. The position of the water front as a function of time was determined from the digitized pictures. The laboratory imagery data and numerical results are quantitatively compared in this study. However, entrapped air-bubble during wave breaking processes cause unavoidable uncertainties (Chan and Melville 1988; Kobayashi and Raichle 1994). Such uncertainties are qualitatively taken into account in the mode-data comparison (see Section 4).

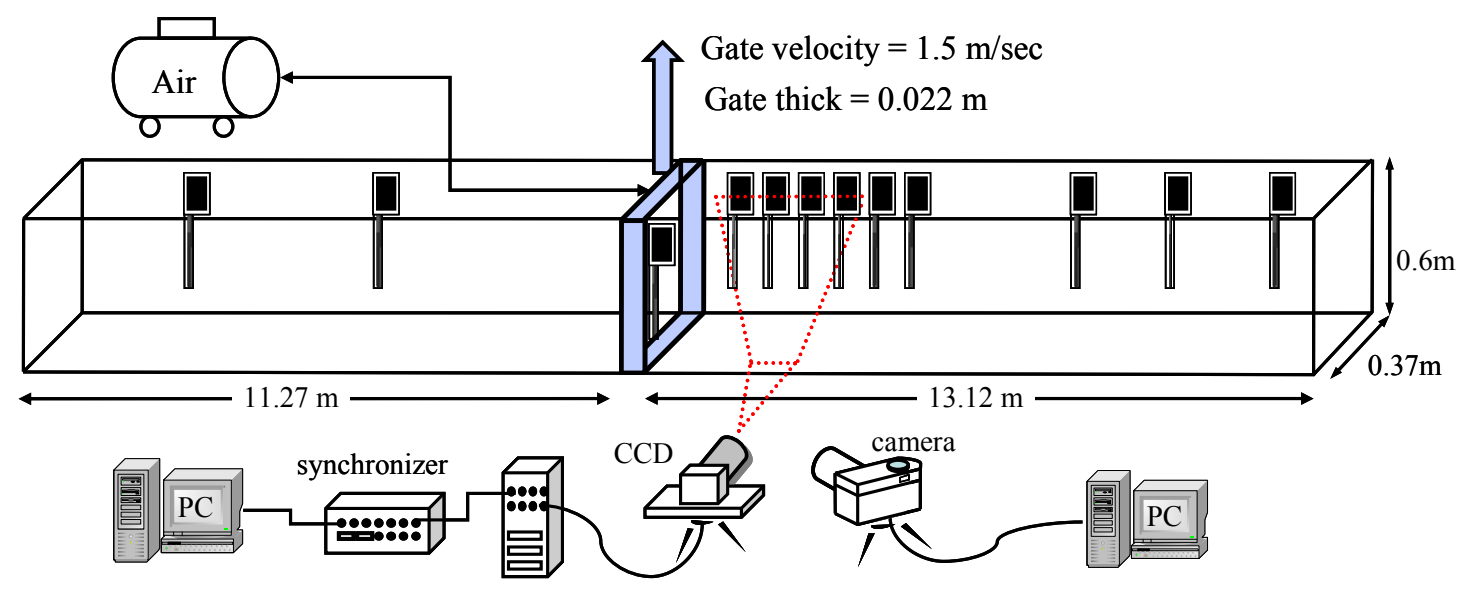

Figure 1. Experimental setup and wave gauges location.

\begin{tabular}{|c|c|c|c|}
\hline \multicolumn{2}{|c|}{ Upstream water depth $(\mathrm{h})=0.3 \mathrm{~m}$} & \multicolumn{2}{|c|}{ Upstream water depth $(\mathrm{h})=0.4 \mathrm{~m}$} \\
\hline Downstream water depth (d) & $r: d / h$ & Downstream water depth (d) & $r: d / h$ \\
\hline $0.015 \mathrm{~m}$ & 0.05 & $0.015 \mathrm{~m}$ & 0.0375 \\
\hline $0.03 \mathrm{~m}$ & 0.1 & $0.04 \mathrm{~m}$ & 0.1 \\
\hline $0.06 \mathrm{~m}$ & 0.2 & $0.08 \mathrm{~m}$ & 0.2 \\
\hline $0.09 \mathrm{~m}$ & 0.3 & $0.12 \mathrm{~m}$ & 0.3 \\
\hline
\end{tabular}




\section{NUMERICAL MODEL}

The numerical model COBRAS (Lin and Liu 1998a, 1998b) is a depth- and time-resolving twodimensional-vertical (2DV) numerical model which solves the Reynolds-Averaged Navier-Stokes (RANS) equations,

$$
\begin{gathered}
\frac{\partial\left\langle u_{i}\right\rangle}{\partial x_{i}}=0 \\
\frac{\partial\left\langle u_{i}\right\rangle}{\partial t}+\left\langle u_{j}\right\rangle \frac{\partial\left\langle u_{i}\right\rangle}{\partial x_{j}}=-\frac{1}{\rho} \frac{\partial\langle p\rangle}{\partial x_{i}}+\frac{1}{\rho} \frac{\partial \tau_{i j}^{v}}{\partial x_{j}}-\frac{\partial\left\langle u_{i}^{\prime} u^{\prime}{ }_{j}\right\rangle}{\partial x_{j}}+g_{i}
\end{gathered}
$$

where $\mathrm{i}, \mathrm{j}=1,2$ for two-dimensional flow, $\left\langle u_{i}\right\rangle$ is the ensemble-averaged flow velocity, $\langle p\rangle$ is the ensemble-averaged fluid pressure, $\tau_{i j}^{v}$ is the viscous stress, $\rho$ is the fluid density, and $g_{i}$ is the gravitational acceleration. The Reynolds stress $-\left\langle u_{i}^{\prime} u_{j}^{\prime}\right\rangle$ is calculated with a nonlinear eddy viscosity relationship suggested by Shih et al. 1996. This nonlinear closure relationship for Reynolds stress mimics the more complete Reynolds-stress closure without solving the transport equations of Reynolds stress. Lin and Liu 1998a, 1998b demonstrate that standard linear eddy viscosity closure tends to over predict the diffusion of turbulence under breaking waves, and the nonlinear relationship suggested above predicts a better turbulence statistics when comparing with laboratory data of breaking wave over slopping beaches (Ting and Kirby 1994).

The eddy viscosity is further calculated with a $k-\varepsilon$ closure:

$$
v_{t}=C_{\mu} \frac{k^{2}}{\varepsilon}
$$

The balance equation s of turbulent kinetic energy, $k$, and the turbulent dissipation rate, $\varepsilon$, are given by

$$
\frac{\partial k}{\partial t}+\frac{\partial k\left\langle u_{j}\right\rangle}{\partial x_{j}}=\frac{\tau_{i j}^{f}}{\rho} \frac{\partial u_{i}}{\partial x_{j}}+\frac{\partial}{\partial x_{j}}\left[\left(v+\frac{v_{t}}{\sigma_{k}}\right) \frac{\partial k}{\partial x_{j}}\right]-\varepsilon+g \frac{v_{t}}{\sigma_{c}}(s-1) \frac{\partial\langle\phi\rangle}{\partial z},
$$

and

$$
\begin{aligned}
\frac{\partial \varepsilon}{\partial t}+\frac{\partial \varepsilon\left\langle u_{j}\right\rangle}{\partial x_{j}} & =C_{\varepsilon 1} \frac{\varepsilon}{k} \frac{\tau_{i j}^{f}}{\rho} \frac{\partial\left\langle u_{i}\right\rangle}{\partial x_{j}}+\frac{\partial}{\partial x_{j}}\left[\left(v+\frac{v_{t}}{\sigma_{\varepsilon}}\right) \frac{\partial \varepsilon}{\partial x_{j}}\right]-C_{\varepsilon 2} \frac{\varepsilon^{2}}{k} \\
& +C_{\varepsilon 3}^{*} \frac{\varepsilon}{k} g \frac{v_{t}}{\sigma_{c}}(s-1) \frac{\partial \phi}{\partial z}
\end{aligned}
$$

The empirical coefficients in Eqs. 3 5 are standard values, i.e., $C_{1 \varepsilon}=1.44, C_{2 \varepsilon}=1.92, \sigma_{\varepsilon}=1.3$, and $\sigma_{k}=1.0$, suggested by Rodi 1980 . The last term in the $\mathrm{k}$ and $\varepsilon$ equations are due to sediment-induced density stratification that can change (mostly attenuate) the carrier flow turbulence (e.g., Hagatun and Eidsvik 1986). Suggested by Umlauf and Burchard 2003 and Rodi 1987, we use $C_{3 \varepsilon}^{*}=0.0$ for stable density stratification and $C_{3 \varepsilon}^{*}=1.0$ when stratification becomes unstable. This formulation is often used for modeling suspended load transport, which is appropriate for dilute flow.

Suspended sediment volumetric concentration $\langle\phi\rangle$ is calculated by mass conservation:

$$
\begin{aligned}
\frac{\partial\langle\phi\rangle}{\partial t}+\frac{\partial\langle\phi\rangle\langle u\rangle}{\partial x}+\frac{\partial\langle\phi\rangle\langle w\rangle}{\partial z} & =\frac{\partial\langle\phi\rangle W_{s}}{\partial z}+\frac{\partial}{\partial z}\left[\left(\frac{v_{t}}{\sigma_{c}}+v\right) \frac{\partial\langle\phi\rangle}{\partial z}\right] \\
& +\frac{\partial}{\partial x}\left[\left(\frac{v_{t}}{\sigma_{c}}+v\right) \frac{\partial\langle\phi\rangle}{\partial x}\right]
\end{aligned},
$$

where the Schmidt number $\sigma_{\mathrm{c}}$ is set to be 1.0. The settling velocity is calculated by the Stokes law:

$$
W_{s}=\frac{\left(\rho^{s}-\rho\right) g}{18 \mu} D_{50}^{2}(1-\langle\phi\rangle)^{n}
$$


where $\rho^{\mathrm{s}}=2650 \mathrm{~kg} / \mathrm{m}^{3}, \mathrm{D}_{50}$ is the grain diameter(Here we use $\mathrm{D}_{50}$ is equal to $0.2 \mathrm{~mm}$ in our model simulation). The dependence of concentration is due to hindered settling with $\mathrm{n}=4.0$.

Standard near-wall modeling is adopted to calculate bed friction velocity $u_{*}$ based on the model results of $\langle u\rangle$ obtained at first grid point above the bed. For sediment transport, we adopt logarithmic law for rough bed:

$$
\frac{\langle u\rangle}{u_{*}}=\frac{1}{\kappa} \ln \left(\frac{30 z}{K_{s}}\right)
$$

with the bed roughness $K_{s}=2 \mathrm{D}_{50}$. Friction velocity obtained from eq. 8 is further used as bed boundary condition for $\langle u\rangle$, turbulence kinetic energy $\mathrm{k}$, and turbulent dissipation rate $\varepsilon$ (Rodi 1980). The friction velocity is also used to calculate the Shields parameter:

$$
\theta=\frac{\rho u_{*}^{2}}{\left(\rho^{s}-\rho\right) g D_{50}}
$$

For the bed boundary condition of suspended sediment concentration, a net flux is specified at any given instant, which consists of an upward suspension flux $E$ and a downward deposition flux $D$. The suspension flux $E$ is related to the reference concentration $\phi_{r}$ by the settling velocity:

$$
E=\phi_{r} W_{s}
$$

Reference concentration formulation of Engelund and Fredsøe 1976 is used to specify the reference concentration:

$$
\phi_{r}(\theta)= \begin{cases}0, & \theta<\theta_{c} \\ \phi_{b} \frac{\theta-\theta_{c}}{0.75-\theta_{c}}, & \theta_{c}<\theta<0.75 \\ \phi_{b}, & \theta>\theta_{c}\end{cases}
$$

with the critical Shields parameter specified to be 0.05 . This formulation is widely used on suspended sand transport (see the textbook of Fredsøe and Deigaard 1992). The deposition flux is calculated directly by its definition with the deposition concentration $\phi_{d}$ obtained from the first few grid points above the bed:

$$
D=\phi_{d} W_{s}
$$

The governing equations are solved by finite difference scheme. Two-step projection method is adopted (Chorin 1969) to calculate the RANS equation. The evolution of free-surface is calculated by the volume of fluid (VOF) method (Hirt and Nichols 1981). Combined upwind and central difference scheme is utilized to calculate the advection terms in the $k-\varepsilon$ equations and sediment concentration equation. More details on the numerical scheme can be found in Kothe et al. 1991.

\section{WAVE FRONT DYNAMICS}

The numerical model is validated with the laboratory observations. Specially, free-surface elevation, wave front velocity are compared with the experimental observations.

\subsection{Free-Surface Elevation}

Free-surface spatio-temporal evolution of two different water depth ratio ( $r=0.05$ and 0.1$)$ during their distinct evolutionary phases, including wave propagation, mushroom jet, breaking, impingement and secondary breaking are shown in Figs. 2-3. Laboratory images (right column), measurement data (open squares), and numerical results (solid line) are plotted together for qualitative/quantitative comparison. Laboratory images are specifically shown to identify regimes where air bubble entrainments are significant. Numerical results generally agree with experiment data. Larger differences occurs in the early stages $(t<0.26 \mathrm{sec})$ of the nearly dry bed case $(r=0.05)$ (see Figs. 2 a- c). The characteristic shape of the front is quite similar to the surge wave mentioned by Stansby et al. 1998. It is found that the numerical results under estimates the speed of the wave front at the early stages $(t<0.26 \mathrm{sec})$. As time progresses, the agreement between numerical and experimental improves. 

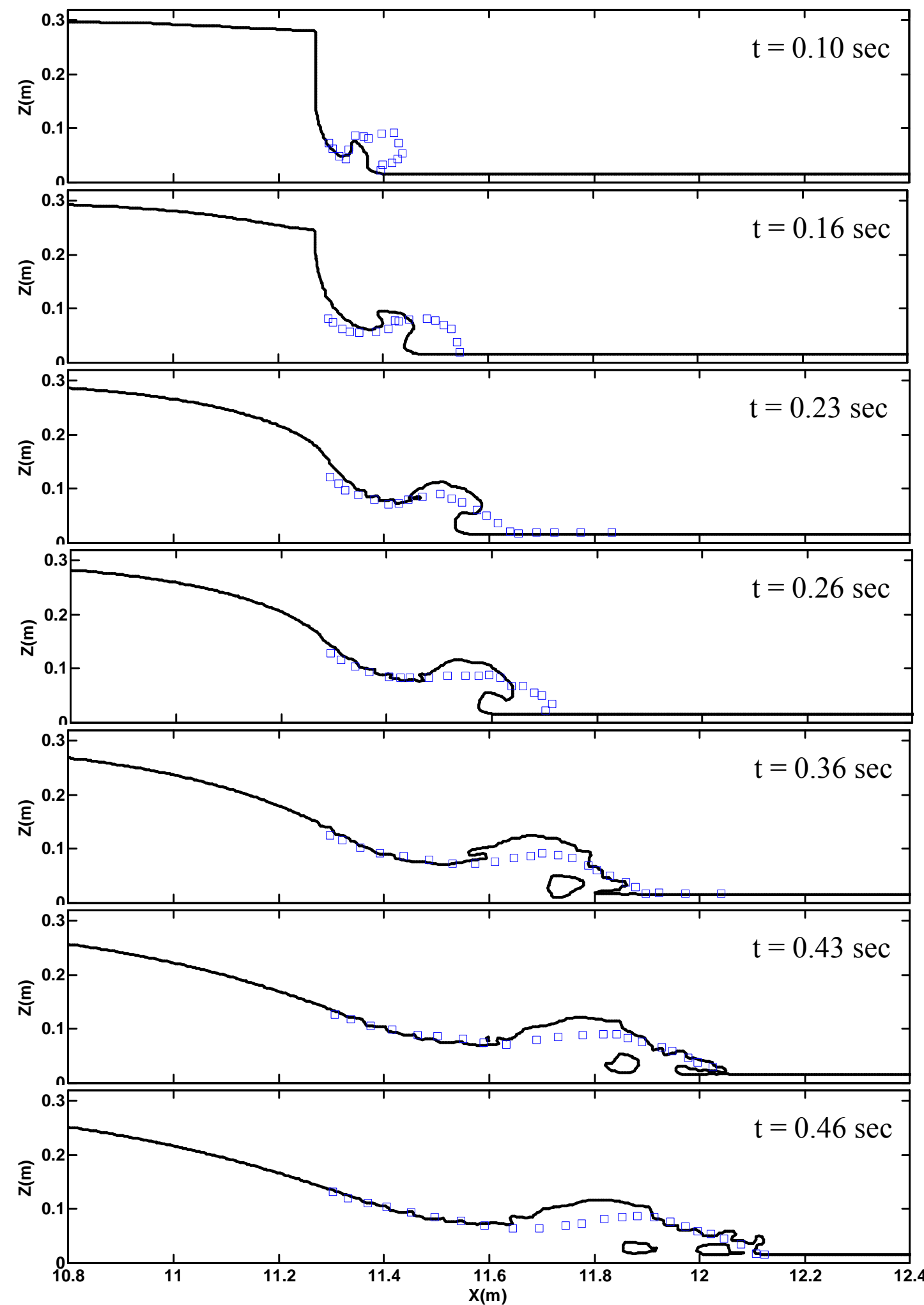

Figure 2. Comparisons of wave front profile between laboratory images (right column), numerical results (left column by solid lines)and measured data (left column by square) at different time intervals for water depth ratio $r=0.05$, upstream water depth $h=0.3 \mathrm{~m}$. 


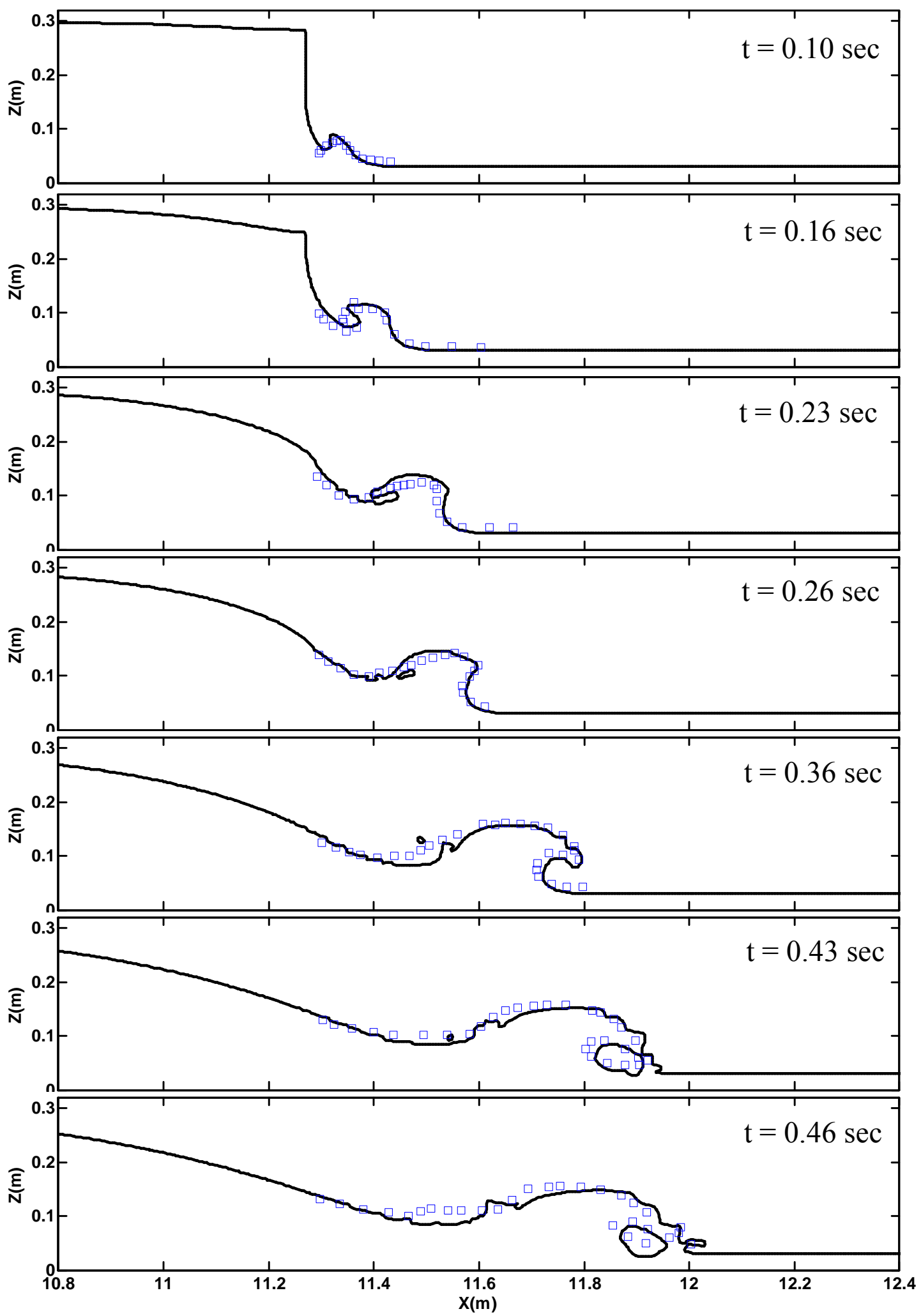

Figure 3. Comparisons of wave front profile between laboratory images (right column), numerical results (left column by solid lines)and measured data (left column by square) at different time intervals for water depth ratio $r=0.1$, upstream water depth $h=0.3 \mathrm{~m}$. 
On the other hand, Fig. 3 shows a better agreement between numerical results and experimental data for the case of larger depth ratio $r=0.1$. The behavior of the wave front is clearly different in the presence of a larger downstream flow depth. A backward jet develops immediately after gate removal and a forward jet is then produced to push the still water downward. As a result, a remarkable mushroom jet forms where a plunging breaker occurs in forward and backward directions. The existence of mushroom jet which shown in Fig. 3 was first reported by Stansby et al. 1998 and Janosi et al. 2004. Although the dam break waves observed here are energetic and highly unsteady, numerical results show very good agreement with experimental data.

\subsection{Wave Front Velocity}

The propagation of the dam break wave is a transient and non-uniform free surface flow with large spatial and temporal gradients, especially at the initial stage of the movement. The behavior of the wave front depends on the water depth ratio $r=d / h$. Fig. 4 shows the comparison of the average velocity of the wave front between the experimental data and numerical results as a function of water depth ratio. The average velocity was computed over two fixed intervals along the first $3 \mathrm{~m}$ of the channel. The experimental results show a decrease in the wave-front velocity with increasing water depth ratio, $r$. The numerical results are also consistent with this trend, showing a good agreement with the experimental data. The fastest wave front velocity is at nearly dry bed case and decreases with $r$. It is noted here that the velocity of the wave front is supercritical and is larger than the reference wave speed, $\sqrt{g h}$ for the water depth ratio smaller than $r=0.2$. For the depth ratio $r$ larger than 0.23 , the wave front velocity is smaller than the reference wave speed and approaches $0.9 \sqrt{g h}$ according to the numerical results of runs with higher $r$ values. In all cases, it also shown that the velocity of the wave front is smaller than the analytical solution, $2 \sqrt{g h}$, (Ritter 1892) due to the finite size of the upstream reservoir in the present experiment. As already discussed in Fig. 2, there are small deviations between the experimental data and numerical results for the cases of nearly dry bed $r \leq 0.1$. However, the difference gradually disappears as the downstream water depth increases.

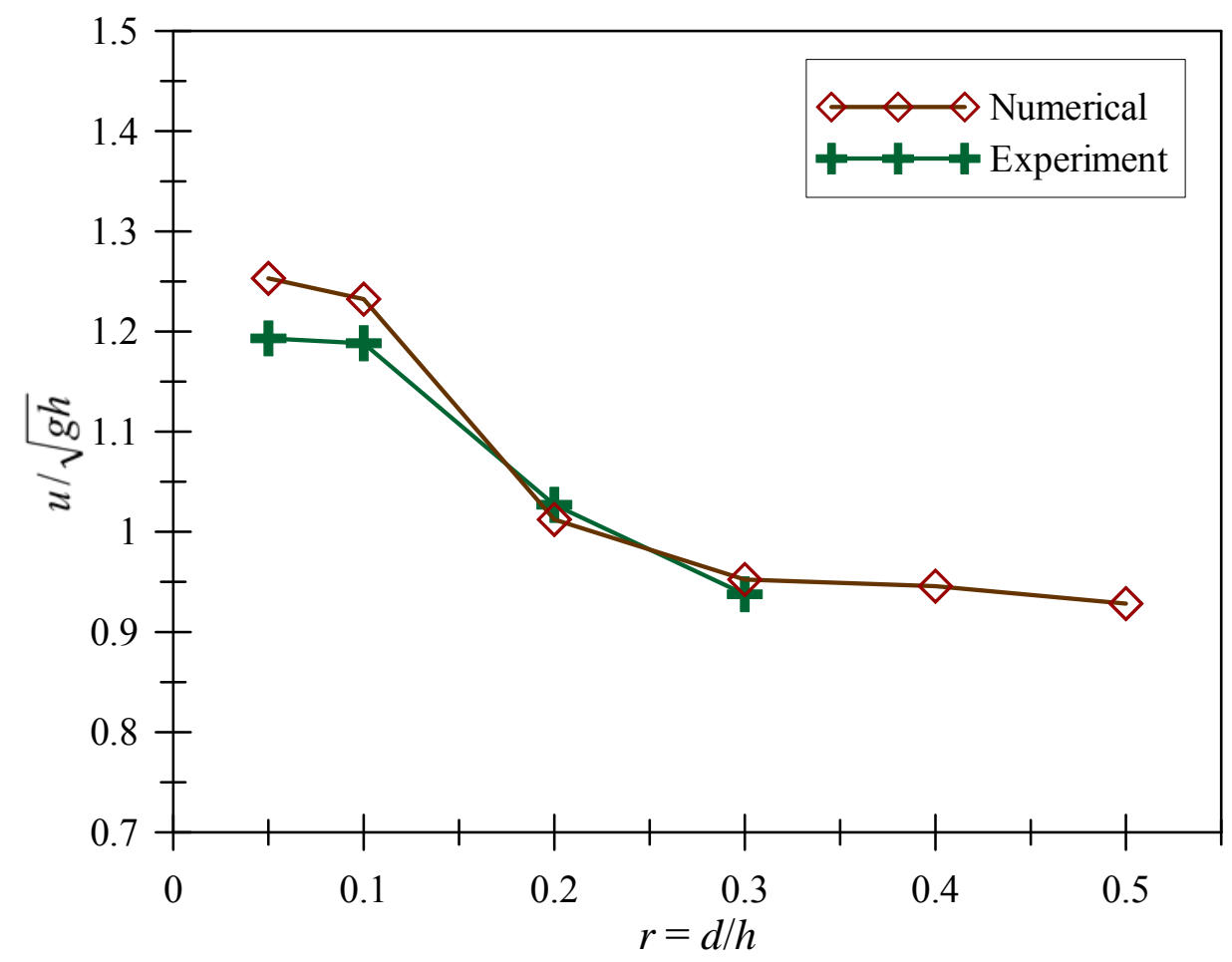

Figure 4. Comparison of the average velocity of the front between the experimental data and numerical results for different downstream-to-upstream water depth ratio $r$. 


\subsection{Turbulence and Sediment Suspension under Dam-Break Waves}

The generation of turbulence due to dam-break waves and the corresponding suspension of sediment are highly dynamic but are closely related. Hence, these two physical quantities are discussed together in this section based on the numerical modeling of the experiments. Under the collapse of dam break flows, turbulence is generated by two mechanisms, i.e., the bottom boundary layer and surface breaking waves. Hence, the downstream flow depth plays an important role in the resulting turbulence generation and sediment transport.

Fig. 5 shows the snapshot of the velocity field and suspended sediment concentration (top panel), turbulent intensity (middle panel), and spatial distribution of Shields parameter and normalized erosion (lower panel) during the very early stage of the dam break $(\mathrm{t}=0.1 \mathrm{sec})$. Once the gate is removed, the flow starts to collapse due to gravity and strong vertical velocity is generated (as large as $-0.9 \mathrm{~m} / \mathrm{s}$ ). Near the bottom, the vertical component of the flow velocity is converted into large horizontal component, which induces bottom stress with a Shields parameter exceeds 2.0 (see red curve in the lower panel in Fig. 5). This high Shields parameter is significantly above the critical value of motion in the sheet flow regime. The turbulence is mainly generated near the bottom. This bottom turbulence along with the high Shields parameter causes the observed high sediment suspension exceeding 100 $\mathrm{g} / \mathrm{L}$ near the initial dam break location. Within this short period of time, the maximum erosion depth is around 3 grain diameter $(0.6 \mathrm{~mm})$, which is located at $\mathrm{x}=11.26 \mathrm{~m}$, very close to the gate location $(\mathrm{x}=11.27 \mathrm{~m})$.
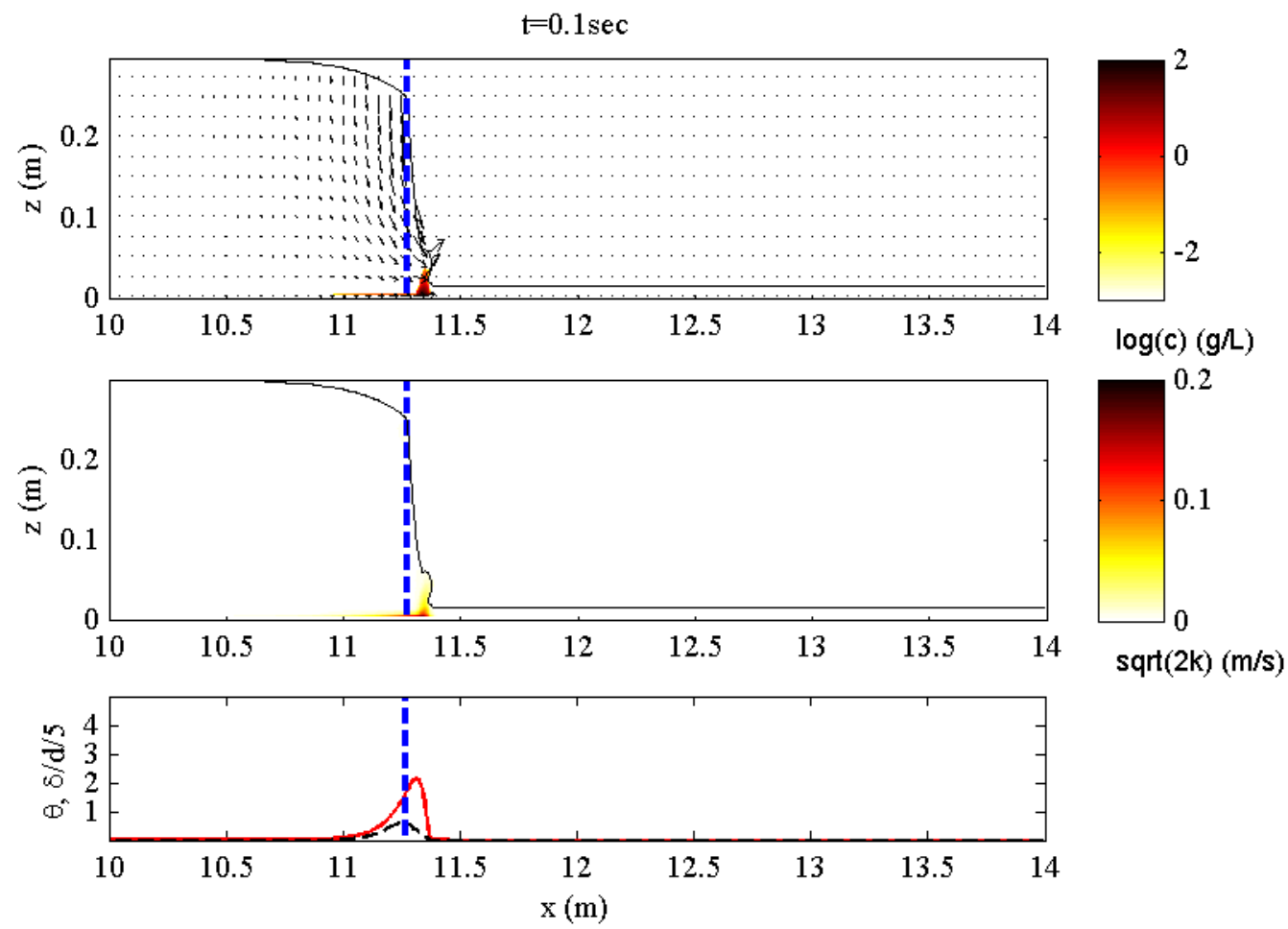

Figure 5. Snapshot of the computed flow field at $\mathrm{t}=0.1 \mathrm{sec}$ for the case of $r=0.05$. Upper panel: Flow velocity and suspended sediment concentration (in logarithmic scale). Middle panel: Turbulent intensity $\sqrt{2 k}(\mathrm{~m} / \mathrm{s})$. Lower panel: streamwise distribution of Shields parameter (solid red curve) and normalized erosion depth $\delta / d$ (divided by 5 , black-dashed curve). The thick dashed line represent the initial gate location $(x=11.27 \mathrm{~m})$.

At $\mathrm{t}=0.35 \mathrm{sec}$ (see Fig. 6), the front of the dam break wave propagates to $\mathrm{x}=11.9 \mathrm{~m}$, where it overturns and plunges. High level of turbulence $\left(2^{\text {nd }}\right.$ panel) is generated both at the breaking waves and the bottom, and it can be clearly observed that these two regimes of turbulence merge into one due to very shallow downstream flow depth. Shields parameter exceeds 3.5 at $\mathrm{x}=11.5 \mathrm{~m}$, which is due contraction of the dam-break flow (flow depth reduces from $0.3 \mathrm{~m}$ to $0.1 \mathrm{~m}$ at $\mathrm{x}=11.5 \mathrm{~m}$ ) that causes large flow velocity and hences bottom stress. More importantly, suspended sediment due to local high bottom stress is further entrained into the breaking wave bore by such high level of turbulence in the water column. Therefore, we clearly see evidence of surface-generated turbulent motion directly 
interaction with bottom sediment suspension, causing high level of sediment suspended ( 100 g/l) throughout the downstream water column. The maximum erosion depth is about $\delta=8 \mathrm{~d}(1.6 \mathrm{~mm})$, which remains located close to the initial gate location $(x=11.2 \mathrm{~m})$. This is because at further upstream, the flow weakens and cannot suspend sufficient amount of sediment to fill in the erosion hole under the initial gate location. On the other hand, although at this instant the location of maximum Shields parameter is located more downstream, there is sufficient amount of sediment coming from the upstream that is deposited there to maintain the scour depth small.
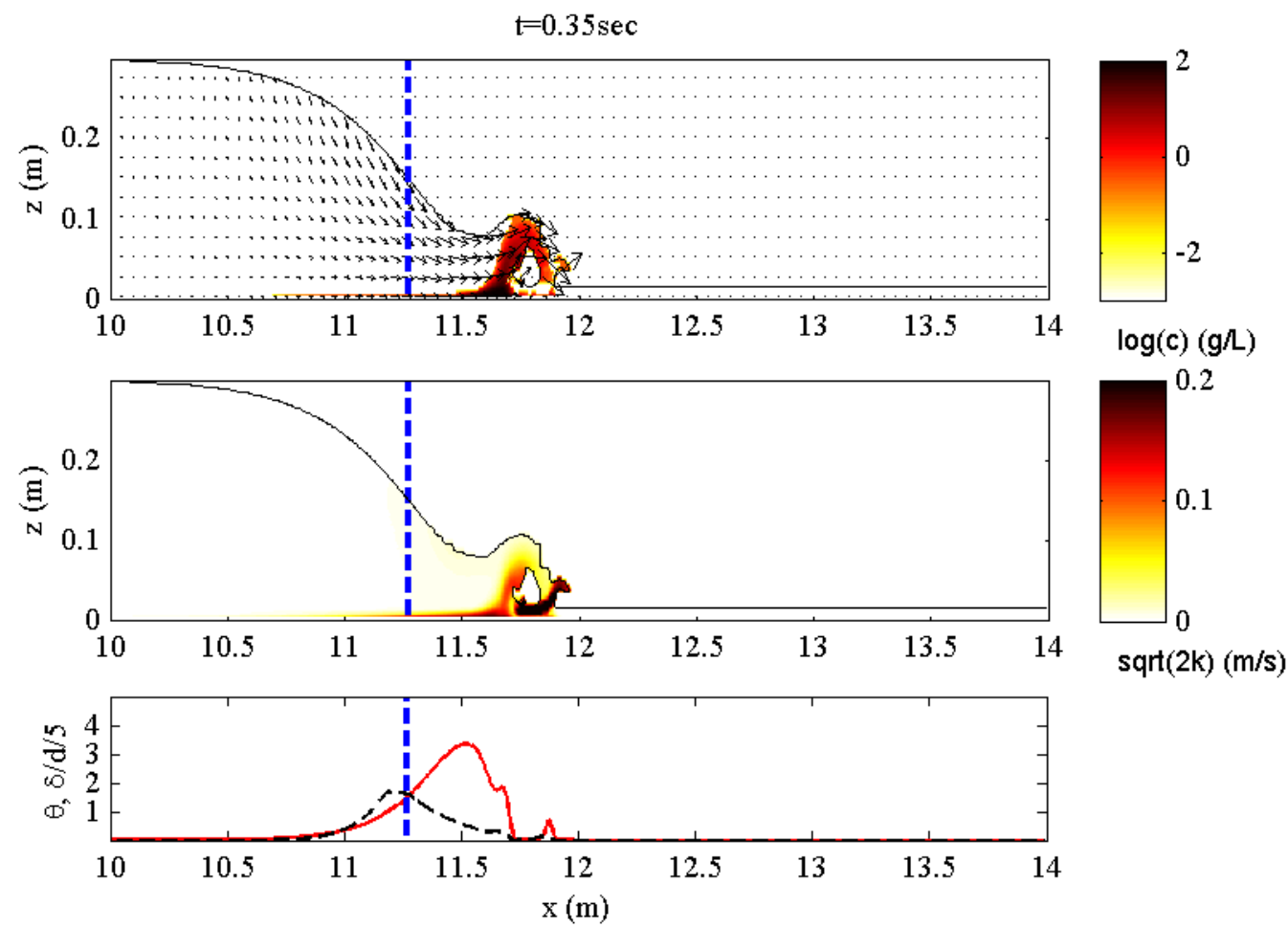

Figure 6. Snapshot of the computed flow field at $\mathrm{t}=0.35 \mathrm{sec}$ for the case of $r=0.05$. Upper panel: Flow velocity and suspended sediment concentration (in logarithmic scale). Middle panel: Turbulent intensity $\sqrt{2 k}(\mathrm{~m} / \mathrm{s})$. Lower panel: streamwise distribution of Shields parameter (solid red curve) and normalized erosion depth $\delta / d$ (divided by 5 , black-dashed curve).

At $\mathrm{t}=0.5 \mathrm{sec}$ (see Fig. 7), the front have plunges twice into the water column. Each plunge generates significant amount of turbulence and allows a large amount of sediment to be suspended from the bed and entrained into the breaking wave bore. Under each plunge, a peak of bottom stress (Shields parameter, see $3^{\text {rd }}$ panel) can be observed. However, the most upstream peak of Shields parameter is still generated by the contraction at $x=11.6 \mathrm{~m}$, which is now weaker (maximum Shields parameter 2.9). The largest erosion depth is still located at $\mathrm{x}=11.2 \mathrm{~m}$ near the initial gate location $(\delta=11 \mathrm{~d})$. Because the present numerical model does not update the bed morphology due to erosion/deposition which has to be further investigated, we can only investigate the initial sediment transport pattern under dam break flow and snapshot of later time is not further examined.

The main characteristics of the turbulent and sediment suspension pattern for the case of $r=0.05$ shown previously is the interaction between the boundary layer and the breaking wave turbulence due to shallow flow depth. Thus, it is also critical to examine such interaction for cases of larger $r$ value. Fig. 8 presents the snapshot at $\mathrm{t}=0.5 \mathrm{sec}$ for the case of $r=0.2$. It is clear that because of the larger flow depth in this case, we observe two hotspots of high level of turbulence, one is near the bed due to bottom friction and the other is located near the surface due to breaking wave (see $2^{\text {nd }}$ panel). These two regimes of high turbulence are detached from each other. The suspended sediment is also confined in the near bed regime and the overall Shields parameter (peak around 2.0) erosion depth is also smaller $(\delta=9 \mathrm{~d})$. 

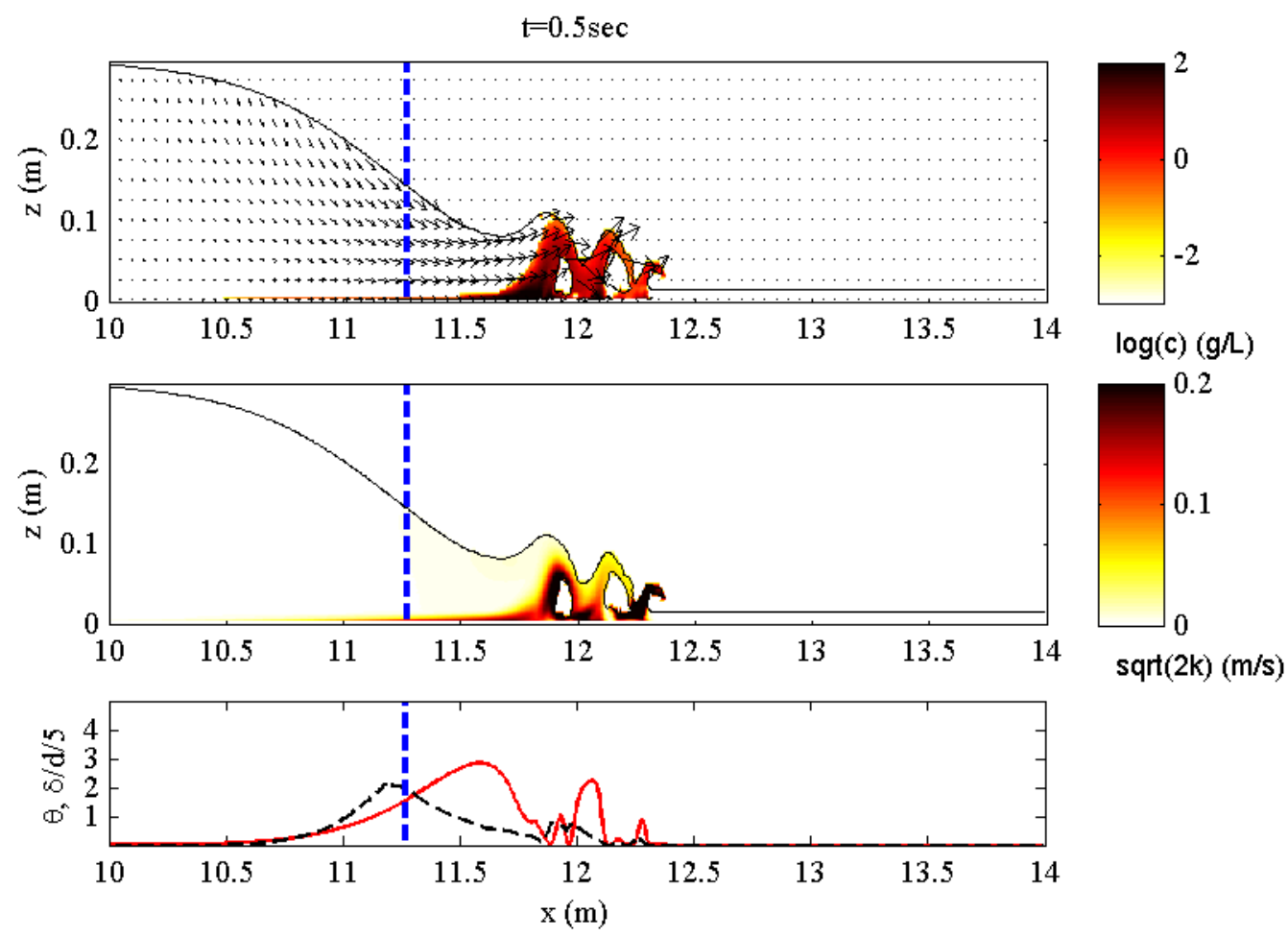

Figure 7. Snapshot of the computed flow field at $\mathrm{t}=0.5 \mathrm{sec}$ for the case of $r=0.05$. Upper panel: Flow velocity and suspended sediment concentration (in logarithmic scale). Middle panel: Turbulent intensity $\sqrt{2 k}(\mathrm{~m} / \mathrm{s})$. Lower panel: streamwise distribution of Shields parameter (solid red curve) and normalized erosion depth $\delta / d$ (divided by 5 , black-dashed curve).
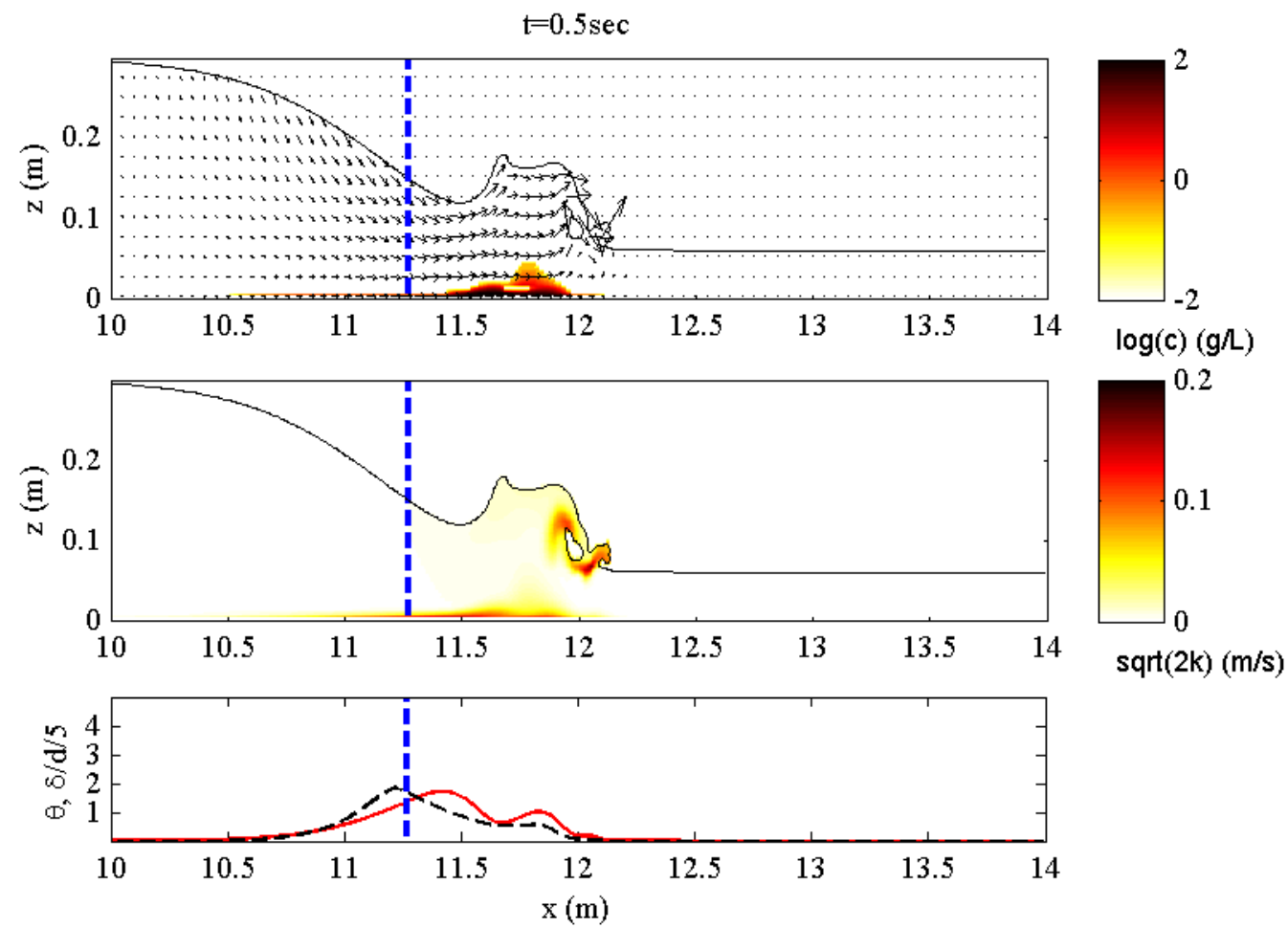

Figure 8. Snapshot of the computed flow field at $t=0.5 \mathrm{sec}$ for the case of $r=0.2)$. Upper panel: Flow velocity and suspended sediment concentration (in logarithmic scale). Middle panel: Turbulent intensity $\sqrt{2 k}(\mathrm{~m} / \mathrm{s})$. Lower panel: streamwise distribution of Shields parameter (solid red curve) and normalized erosion depth $\delta / d$ (divided by 5 , black-dashed curve). 


\section{CONCLUSIONS}

The two-dimensional RANS equations with $k-\varepsilon$ turbulence closure model (COBRAS) have been proved to be a suitable model to describe the complex evolution of dam-break flows with strong spatial and temporal gradients. Experimental wave profiles and horizontal velocities can be suitable simulated by the present model.

Based on the numerical results shown in this paper, as well as the experimental observations, the process of the dam-break propagation can be described in two distinct flow regimes. An initial regime developed in the first instant following dam releasing. In this region, the wave velocity is faster than those for longer distances whereas the increasing bottom friction and breaking decrease the velocity. The difference in these two regimes is higher than the increasing downstream water depth. Depending on the ratio between the downstream and the upstream water depth, the dam-break flow can behave only a forward plunging jet or a mushroom jet.

The behavior of the wave front will depend on the different depth ratio $(r=h / d)$. The numerical and experimental results show a decrease in the wave-front velocity with increasing water depth ratio. The fastest wave front velocity is at nearly dry bed case and decreases with $r$. It is important to point out that the velocity of the wave front is supercritical and is larger than the reference wave speed, $\sqrt{g h}$ for the water depth ratio smaller than $r=0.2$. For the depth ratio $r$ larger than 0.23 , the wave front velocity is smaller than the reference wave speed and is approached to $0.9 \sqrt{g h}$ according to the numerical results. A maximum value of the forward breaking jet velocity occurs at the water depth ratio $r=0.1$. The forward breaking jet velocity will decrease for higher water depth ratio. There is a linear relationship between the difference of the water level in the upstream and downstream and the depth ratio. The numerical results show that an increase in the displacement of the breaking jet from the gate with increasing depth ratio $r$.

\section{ACKNOWLEDGMENTS}

The authors would like to acknowledge the financial support from National Science Council of Taiwan under grant no. 99-2923-E-110-001.

\section{REFERENCES}

Abderrezzak, K.E.K., A. Paquier, and B. Gay. 2008. One-dimensional numerical modelling of dambreak waves over movable beds: application to experimental and field cases, Environmental Fluid Mechanics, 8(2), 169-198.

Capart, H., and D.L. Young. 1998. Formation of a jump by the dam-break wave over a granular bed, Journal of Fluid Mechanics, 372, 165-187.

Chan, E.S., and W.K. Melville. 1988. Deep-water plunging wave pressures on a vertical plane wall, Proceedings of the Royal Society of London Series a-Mathematical Physical and Engineering Sciences; 417(1852), 95-131.

Cheng, M.Y., and L. Peng. 2006. Simple and efficient improvements of multivariate local linear regression, Journal of Multivariate Analysis, 97(7), 1501-1524.

Chorin, A.J. 1969. On Convergence of discrete approximations to Navier-Stokes equations, Mathematics of Computation, 23(106), 341-353.

Engelund, F., and J. Fredsøe. 1976. Sediment transport model for straight alluvial channels, Nordic Hydrology, 7(5), 293-306.

Fraccarollo, L., and H. Capart. 2002. Riemann wave description of erosional dam-break flows, Journal of Fluid Mechanics, 461, 183-228.

Fredsøe, J., and R. Deigaard. 1992. Mechanics of Coastal Sediment Transport, Advanced Series on Ocean Engineering, Vol. 3, World Scientific.

Hagatun, K., and K.J. Eidsvik. 1986. Oscillating turbulent boundary-layer with suspended sediments, Journal of Geophysical Research-Oceans, 91(C11), 3045-3055.

Hirt, C.W., and B.D. Nichols. 1981. Volume of fluid (Vof) method for the dynamics of free boundaries, Journal of Computational Physics, 39(1), 201-225.

Huang, J.J., W.Y. Hsu, R.Y. Yang, and H.H. Hwung. 2010. The experiment of flood bore propagation of dam-break, Proceeding of the 32nd Ocean Engineering Conference in Taiwan National Taiwan Ocean University, 587-592.

Janosi, I.M., D. Jan, K.G. Szabo, and T. Tamas. 2004. Turbulent drag reduction in dam-break flows, Experiments in Fluids, 37(2), 219-229. 
Kao, S.J., and J.D. Milliman. 2008. Water and sediment discharge from small mountainous rivers, Taiwan: The roles of lithology, episodic events, and human activities, Journal of Geology, 116(5), 431-448.

Kobayashi, N., and A.W. Raichle. 1994. Irregular wave overtopping of revetments in surf zones, Journal of Waterway Port Coastal and Ocean Engineering-Asce, 120(1), 56-73.

Kothe, D.B., R.C. Mjolsness and M.D. Torrey. 1991. RIPPLE: A Computer Program for Incompressible Flows with Free Surfaces, Los Alamos National Lab., LA-12007-MS, Los Alamos, New Mexico.

Lin, P.Z., and P.L.F. Liu. 1998a. A numerical study of breaking waves in the surf zone, Journal of Fluid Mechanics, 359, 239-264.

Lin, P.Z., and P.L.F. Liu. 1998b. Turbulence transport, vorticity dynamics, and solute mixing under plunging breaking waves in surf zone, Journal of Geophysical Research-Oceans, 103(C8), 1567715694.

Ritter, A. 1892. Die Fortpflanzung de Wasserwellen, Zeitschrift Verein Deutscher Ingenieure, 36(33), 47-954.

Rodi, W. 1980. Turbulence models and their application in hydraulics, International Association of Hydraulic Engineering (IAHR) Monograph, Delft, The Netherlands.

Rodi, W. 1987. Examples of calculation methods for flow and mixing in stratifled fluids, J. Geophys. Res., 92, 5305-5328.

Shih, T.H., J. Zhu, and J. Lumley. 1996. Calculation of wall-bounded complex flows and free shear flows, International Journal for Numerical Methods in Fluids, 23(11), 1133-1144.

Stansby, P.K., A. Chegini, and T. Barnes. 1998. The initial stages of dam-break flow, Journal of Fluid Mechanics, 370, 203-220.

Ting, F.C.K., and J.T. Kirby. 1994. Observation of undertow and turbulence in a laboratory surf zone, Coastal Engineering, 24(1-2), 51-80.

Umlauf, L., and H. Burchard. 2003. A generic length-scale equation for geophysical turbulence models, Journal of Marine Research, 61(2), 235-265.

Water Resources Agency, Ministry of Economic Affairs. 2009. The storm rainfall and flood during typhoon Morakot.

Wu, W., and S.S.Y. Wang. 2007. One-dimensional modeling of dam-break flow over movable beds, $J$ Hydr Eng, 133(1), 48-58. 